\title{
Cogitare
Enfermagem
}

\section{QUALIDADE DE VIDA E EXPERIÊNCIAS RELIGIOSAS/ ESPIRITUAIS DE PACIENTES COM CÂNCER EM UM SERVIÇO DE EMERGÊNCIA}

Carla Roberta Monteiro Miuraํㅜ, Cássia Regina Vancini Campanharo², Ruth Ester Assayag Batista ${ }^{3}$, Maria Carolina Barbosa Teixeira Lopes ${ }^{4}$, Priscila Fernandes Barros ${ }^{5}$, Meiry Fernanda Pinto Okuno ${ }^{6}$

\section{RESUMO}

Objetivo: avaliar qualidade de vida, experiências espirituais em pacientes com câncer e sua correlação.

Método: estudo transversal, realizado na Emergência, de fevereiro a setembro de 2017. Utilizado - Medical Outcome Study 36 - Item Short-Form Health Survey para verificação da qualidade de vida, com oito dimensões, escore de 0 (pior estado) a 100 (melhor estado) e a Escala Diária de Experiência Espiritual, com 16 itens, pontuação entre 16 e 94 (menores pontuações refletem maior frequência de experiências). Utilizou-se o Coeficiente de Spearman para correlacionar qualidade de vida e espiritualidade.

Resultados: 83 pacientes, média de idade 58 anos, predomínio masculino. A dimensão da qualidade de vida mais comprometida foi "aspecto físico". A Escala de Experiência Espiritual apresentou média 51, com correlação negativa entre qualidade de vida e espiritualidade.

Conclusão: a espiritualidade relaciona-se com qualidade de vida e deve ser considerada no atendimento a pacientes com câncer na emergência.

DESCRITORES: Perfil de Impacto da Doença; Religião; Enfermagem em Emergência; Oncologia; Espiritualidade.

\section{COMO REFERENCIAR ESTE ARTIGO:}

Miura CRM, Campanharo CRV, Batista REA, Lopes MCBT, Barros PF, Okuno MFP. Qualidade de vida e experiências religiosas/espirituais de pacientes com câncer em um serviço de emergência. Cogitare enferm. [Internet]. 2020 [acesso em "colocar data de acesso, dia, mês abreviado e ano"]; 25. Disponível em: http://dx.doi.org/10.5380/ ce.v25i0.67474.

Este obra está licenciado com uma Licença Creative Commons Atribuição 4.0 Internacional.

${ }^{1}$ Enfermeira. Doutora em Enfermagem. Docente de Enfermagem da Universidade Federal de São Paulo. São Paulo, SP, Brasil. $\odot$

${ }^{2}$ Enfermeira. Doutora em Enfermagem. Docente de Enfermagem da Universidade Federal de São Paulo. São Paulo, SP, Brasil. $\odot$

${ }^{3}$ Enfermeira. Doutora em Enfermagem. Docente de Enfermagem da Universidade Federal de São Paulo. São Paulo, SP, Brasil. $\bigcirc$

${ }^{4}$ Enfermeira. Doutora em Enfermagem. Docente de Enfermagem da Universidade Federal de São Paulo. São Paulo, SP, Brasil.

${ }^{5}$ Enfermeira. Especialista em Urgência e Emergência. Hospital Euryclides de Jesus Zerbini. São Paulo, SP, Brasil. $\odot$

${ }^{6}$ Enfermeira. Doutora em Enfermagem. Docente de Enfermagem da Universidade Federal de São Paulo. São Paulo, SP, Brasil. $\odot$ 


\title{
QUALITY OF LIFE AND RELIGIOUS/SPIRITUAL EXPERIENCES OF CANCER PATIENTS IN AN EMERGENCY SERVICE
}

\author{
ABSTRACT \\ Objective: Evaluate the quality of life, spiritual experiences in cancer patients and their \\ correlation. \\ Method: Cross-sectional study, carried out in the Emergency, from February to September \\ 2017. Using the Medical Outcome Study 36 - Item Short-Form Health Survey for quality \\ of life verification, with eight dimensions, the scores from 0 (worst condition) to 100 (best \\ condition) and the Daily Scale of Spiritual Experience, with 16 items, scoring between 16 and \\ 94 (lower scores reflect higher frequency of experience). The Spearman coefficient was used \\ to correlate quality of life and spirituality. \\ Results: 83 patients, mean age 58 years, male predominance. The most compromised quality \\ of life's dimension of was "physical aspect". The Spiritual Experience Scale presented a mean \\ of 51, with a negative correlation between the quality of life and spirituality. \\ Conclusion: Spirituality is related to quality of life and should be considered in the care of \\ cancer patients in the emergency.
}

DESCRIPTORS: Disease Impact Profile; Religion; Emergency Nursing; Oncology; Spirituality.

\section{CALIDAD DE VIDA Y EXPERIENCIAS RELIGIOSAS/ESPIRITUALES DE PACIENTES CON CANCER EN UM SERVICIO DE EMERGENCIA}

\author{
RESUMEN: \\ Objetivo: evaluar la correlación entre la calidad de vida y las experiencias espirituales en \\ pacientes con cáncer. \\ Método: estudio transversal, realizado en el Departamento de Emergencias, de febrero a \\ septiembre de 2017. Par verificar la calidad de vida se utilizó el Medical Outcome Study 36 \\ - Item Short-Form Health Survey, con ocho dimensiones, puntaje de 0 (peor estado) a 100 \\ (mejor estado) y la Escala Diaria de Experiencia Espiritual, con 16 ítems, puntajes entre 16 y \\ 94 (los puntajes más bajos reflejan una mayor frecuencia de experiencias). El coeficiente de \\ Spearman se utilizó para correlacionar la calidad de vida y la espiritualidad. \\ Resultados: el estudio se realizó con 83 pacientes, edad promedio 58 años, predominantemente \\ hombres. La dimensión de calidad de vida más comprometida fue el "aspecto físico". La \\ escala de experiencia espiritual mostró un promedio de 51, con una correlación negativa \\ entre calidad de vida y espiritualidad. \\ Conclusión: la espiritualidad está relacionada con la calidad de vida y debe considerarse en la \\ atención de pacientes con cáncer en la sala de emergencias.
}

DESCRIPTORES: Perfil de Impacto de la Enfermedad; Religión; Enfermería de Emergencia; Oncología; Espiritualidad. 
Câncer é a denominação de um conjunto de doenças que se caracterizam pela multiplicação das células de forma desordenada, por mutação no DNA celular, em algum tecido ou órgão do corpo, e possuem capacidade de invadir outros sistemas do organismo através de vasos sanguíneos, num processo chamado de metástase ${ }^{(1,2)}$. É um problema de saúde pública reconhecido pela Organização Mundial de Saúde (OMS), sendo a segunda maior causa de morte no Brasil, com estimativa de mais de 600 mil novos casos para cada ano do biênio 2018-2019(1-4).

Pacientes com câncer experimentam pelo menos uma emergência durante a sua doença e o desenvolvimento de novos tratamentos e estratégias para pacientes oncológicos tem resultado em uma vida prolongada e um aumento do número de emergências que possam ser vivenciadas em decorrência destes novos tratamentos ${ }^{(5)}$.

As emergências oncológicas podem ser categorizadas como metabólicas (hipercalcemia, Síndrome da Secreção Inapropriada de Hormônio Antidiurético, Síndrome de lise tumoral), hematológicas (neutropenia febril), estruturais (compressão de nervos periféricos ou medula espinhal, derrame pericárdico maligno) ou efeitos colaterais de agentes quimioterápicos (diarreia, extravasamento) ${ }^{(5,6)}$.

A unidade de emergência requer cuidados de alta complexidade no atendimento a pacientes em situação de risco iminente de vida. Os profissionais deste setor vivem diariamente sob a pressão ocasionada pela necessidade do ganho de tempo, rapidez e precisão, pela elevada demanda de atendimentos e pelas experiências diárias de morte e sofrimento. Torna-se, pois, neste cenário, um desafio para a enfermagem a construção de seu fazer, considerando todas as dimensões do cuidado(6).

A assistência humanizada visa assistir o ser humano em sua integralidade, ocupandose tanto dos componentes adoecidos quanto dos sadios do ser, tais como a espiritualidade/ religiosidade.

Espiritualidade e religião são consideradas popularmente sinônimos, porém suas definições são distintas. A primeira possui um conceito amplo e pessoal, é a maneira como o indivíduo se relaciona com os outros, com a natureza e com o universo, além de ser a busca por respostas sobre o significado e propósito da vida; é a capacidade de lidar com sentimentos e frustrações. Já a religião é um conjunto de crenças organizadas e associadas a práticas ritualísticas, seguindo normas e dogmas de uma doutrina, compartilhado por uma comunidade para aproximá-los daquilo que é sagrado, divino(4-7).

A espiritualidade pode ser uma estratégia de enfrentamento do paciente perante o câncer, atribuindo significado ao processo de adoecimento e sofrimento ${ }^{(8,9)}$. É importante ressaltar que a prática espiritual/religiosa, no contexto do tratamento de doenças crônicas, deve existir junto à prática da medicina. A melhora da saúde mental proporcionada pela fé possibilita o aumento da adesão ao tratamento médico(7).

São estabelecidos pela OMS que a religiosidade e a espiritualidade são conceitos que compõem a Qualidade de vida $(\mathrm{QV})^{(10)}$. Estudo de revisão sobre qualidade de vida relacionada à saúde de pacientes com câncer avançado mostrou resultados que intervenções espirituais podem promover significativa melhora das manifestações clínicas e $\mathrm{QV}^{(11)}$. Entre os efeitos benéficos das práticas religiosas/espirituais, estão a redução de estresse e depressão, melhor controle da pressão arterial, redução do uso de álcool ou outras substâncias, maior nível de bem-estar, otimismo e esperança ${ }^{(12)}$.

Diante do aumento do número de pacientes com câncer no Brasil e do consequente crescimento da procura pelo Serviço de Emergência por essa população, faz-se fundamental o conhecimento acerca da OV e de sua relação com a religiosidade/espiritualidade do paciente oncológico em situação de emergência, com vistas a uma assistência integral e acolhedora neste cenário de prática profissional. Além disso, estudos que tratem dessa 
temática na emergência ainda são escassos.

Diante do exposto, este estudo teve como objetivo avaliar a qualidade de vida, mensurar a frequência de experiências religiosas/espirituais em pacientes com diagnóstico de câncer atendidos em um serviço de emergência, e verificar sua correlação.

\section{MÉTODO}

Trata-se de um estudo epidemiológico, transversal e analítico, realizado no serviço de emergência de um hospital de ensino da cidade de São Paulo, no período de fevereiro a setembro de 2017. Foram incluídos no estudo 83 pacientes que atenderam aos critérios de inclusão: idade acima de 18 anos, diagnóstico prévio de câncer, estar sem desconforto respiratório e instabilidade hemodinâmica no momento da coleta de dados, e aceitar participar livremente da pesquisa.

O cálculo amostral foi realizado pelo método de amostragem probabilística estratificada proporcional ao número médio de pacientes a partir de 18 anos, que procuraram atendimento no Serviço de Emergência do hospital do estudo nos seis meses que antecederam a pesquisa. O cálculo considerou um grau de confiança maior ou igual a $80 \%$ e alfa de $5 \%$, com base nas características idade, gênero, escolaridade, estado civil, ocupação, religião, rede de apoio, cor e renda familiar. O resultado indicou a necessidade de incluir 80 pacientes para alcançar os objetivos propostos.

Para o levantamento de dados sociodemográficos, aplicou-se um questionário estruturado elaborado pelos pesquisadores. A fim de avaliar a QV, foi aplicado o instrumento Medical Outcome Study 36 - Item Short-Form Health Survey (SF-36) ${ }^{(12)}$, traduzido e validado no Brasil. O SF-36 é um questionário genérico composto por oito dimensões (capacidade funcional, aspecto físico, dor, estado geral de saúde, vitalidade, aspecto social, aspecto emocional e saúde mental), e o escore de cada dimensão varia de 0 (pior estado de saúde) a 100 (melhor estado de saúde)(12). Para mensurar a frequência de experiências religiosas/espirituais, foi utilizada a Escala Diária de Experiência Espiritual(12) . Ela avalia a frequência com que as pessoas vivenciam, no seu cotidiano, experiências como a sensação da presença de Deus, fortaleza e conforto na religião ou na espiritualidade, conexão com a vida de modo geral, amor aos outros, admiração pela natureza, paz interior, gratidão por bênçãos e desejo de proximidade com Deus. É composta por 16 itens e é considerada uma medida unidimensional. O escore total é obtido pela soma das pontuações dos 16 itens, podendo variar de 16 a 94. Menores pontuações refletem maior frequência de experiências religiosas/espirituais ${ }^{(13)}$.

As variáveis categóricas foram apresentadas por meio de tabelas em suas frequências absolutas e relativas, e as variáveis contínuas por meio da média, desvio padrão, mediana, mínimo e máximo.

Para verificar a relação entre QV e Experiências religiosas/espirituais, foi utilizado o Coeficiente de Correlação de Spearman. O programa utilizado para a análise foi o Statistical Package for the Social Sciences versão 19. O nível de significância adotado foi de $5 \%$ e foram consideradas significativas as estatísticas com p descritivo menor ou igual a 0,05.

Os dados foram coletados mediante aprovação do Comitê da Ética da Universidade Federal de São Paulo (Parecer $n^{\circ}$ 1.903.796 /CAAE $n^{\circ}$ 63925317.4.0000.5505) e assinatura do termo de consentimento livre e esclarecido pelos participantes. A pesquisa foi desenvolvida dentro dos padrões éticos descritos. 
A amostra foi composta por 83 pacientes com idade média de 58 anos, variando de 25 a 84 anos, sendo $54,2 \%$ do sexo masculino, $46,9 \%$ casados e $33,7 \%$ da cor negra. Dentre os pacientes envolvidos no estudo, $42(50,6 \%)$ eram pensionistas/aposentados, 36 $(43,3 \%)$ referiram ter cursado até o ensino fundamental e $28(33,7 \%)$ possuir renda familiar de até dois salários mínimos, conforme apresentado na Tabela 1.

Tabela 1 - Dados sociodemográficos e econômicos dos pacientes com diagnóstico de câncer atendidos no Serviço de Emergência. São Paulo, SP, Brasil, 2017 (continua)

\begin{tabular}{lc}
\hline Variáveis & $\mathbf{n}(\%)$ \\
\hline Idade & $58,1(12,4)$ \\
\hline Média (DP) & $60(25-84)$ \\
\hline Mediana (Mínimo-Máximo) & \\
\hline Sexo & $45(54,2)$ \\
\hline Masculino & $38(45,7)$ \\
\hline Feminino & \\
\hline Estado civil & $39(46,9)$ \\
\hline Casado & $19(22,8)$ \\
\hline Viúvo & $15(18,0)$ \\
\hline Divorciado/separado & $10(12,0)$ \\
\hline Solteiro & \\
\hline Cor & $28(33,7)$ \\
\hline Negra & $27(32,5)$ \\
\hline Branca & $26(31,3)$ \\
\hline Parda & $2(2,4)$ \\
\hline Amarela & \\
\hline Ocupação & $42(50,6)$ \\
\hline Pensionista/aposentado & $16(19,2)$ \\
\hline Empregado & $15(18,0)$ \\
\hline Desempregado & $10(12,0)$ \\
\hline Do lar & $28(33,7)$ \\
\hline Escolaridade & $25(30,1)$ \\
\hline Ensino fundamental & $17(20,4)$ \\
\hline Ensino médio & $26(31,3)$ \\
\hline Ensino superior & $18(21,6)$ \\
\hline Não letrado & $3(3,6)$ \\
\hline Renda familiar & \\
\hline Até 1 SM & \\
\hline 2 SM & \\
\hline 3 SM & \\
\hline 4 SM & \\
\hline & \\
\hline
\end{tabular}




\begin{tabular}{lc}
\hline $5 \mathrm{SM}$ & $2(2,4)$ \\
\hline Número de dependentes da renda & \\
\hline Média (DP) & $2,3(0,9)$ \\
\hline Mediana (Mínimo-Máximo) & $2(1-4)$ \\
†SM- Salário Mínimo (valor= R\$937,00) &
\end{tabular}

A Tabela 2 apresenta as características clínicas dos pacientes que compuseram a amostra. O tipo de câncer mais frequente foi o de sistema reprodutor, seguido do gastrointestinal. $66(79,5 \%)$ pacientes tinham comorbidades, sendo prevalente a cardiopatia 14 (69\%). Entre os pacientes participantes, 81 (97,5\%) faziam uso de medicamentos contínuos, sendo a classe mais frequente a dos analgésicos 49 (49\%). A média do tempo de internação foi de 1,7 dia, variando de um a sete dias.

Tabela 2 - Características clínicas dos pacientes com diagnóstico de câncer atendidos no Serviço de Emergência. São Paulo, SP, Brasil, 2017

\begin{tabular}{lc} 
Variáveis & $\mathbf{n}(\%)$ \\
\hline Tipos de neoplasia (sistemas) & $19(22,9)$ \\
\hline Reprodutor & $18(21,6)$ \\
\hline Gastrointestinal & $9(10,8)$ \\
\hline Sanguíneo & $7(8,43)$ \\
\hline Linfático & $7(8,43)$ \\
\hline Respiratório & $11(13,2)$ \\
\hline Urinário & $4(4,82)$ \\
\hline Pele & $4(4,81)$ \\
\hline Endócrino & $2(2,41)$ \\
\hline Nervoso central & $2(2,41)$ \\
\hline Músculo esquelético & \\
\hline Comorbidades & $28(33,7)$ \\
\hline Cardiopatia & $27(32,5)$ \\
\hline Hipertensão Arterial & $26(31,3)$ \\
\hline Diabetes Mellitus & $2(2,4)$ \\
\hline Nefropatia & $10(12,0)$ \\
\hline Doenças respiratórias & $26(31,3)$ \\
\hline Medicamentos em uso & $36(43,3)$ \\
\hline Analgésico & $16(19,2)$ \\
\hline Anti-hipertensivo & $15(18,0)$ \\
\hline Hipoglicemiante & \\
\hline Protetor gástrico & 126 \\
\hline Hipolipemiante & 10 \\
\hline
\end{tabular}


Dos entrevistados, $74(89,1 \%)$ possuíam alguma religião, com predomínio da católica $30(40,5 \%)$, seguida pela evangélica $29(39,1 \%)$.

Dentre os participantes, $45(54,2 \%)$ referiram não possuir qualquer rede de apoio. Unidades Básicas de Saúde (UBS) foram as mais citadas $16(42,1 \%)$ por aqueles que referiram possuir apoio. A igreja foi citada como rede de apoio combinada à UBS por cinco $(13,1 \%)$ participantes.

Verifica-se na Tabela 3 que as dimensões da qualidade de vida que se mostraram mais comprometidas foram as dimensões de "Aspecto físico" $(23,1)$, seguida pela "Estado Geral de Saúde" $(28,1)$ e "Capacidade Funcional" $(38,3)$. Para a Escala Diária de Experiência Espiritual, os envolvidos no estudo apresentaram uma média de escore de 51.

Tabela 3 - Qualidade de vida (SF-36) e frequência de experiências religiosas/espirituais de pacientes com diagnóstico de câncer atendidos no Serviço de Emergência. São Paulo, SP, Brasil, 2017

\begin{tabular}{lc} 
Dimensões SF-36 & Média (DP) \\
\hline Capacidade funcional & $38,3(21,7)$ \\
\hline Aspecto físico & $23,1(26,4)$ \\
\hline Dor & $43,8(14,2)$ \\
\hline Estado geral de saúde & $28,1(9,2)$ \\
\hline Vitalidade & $40,5(14)$ \\
\hline Aspectos sociais & $44,3(16,7)$ \\
\hline Aspectos emocionais & $39,2(32,6)$ \\
\hline Escala de Experiência religiosa/ espiritual & $51(13,7)$
\end{tabular}

Verificou-se correlação negativa estatisticamente significante entre todas as dimensões da SF-36 e o escore da Escala Diária de Experiência Espiritual. Portanto, quanto menor a frequência de experiências religiosas/espirituais, pior o escore da QV, como se observa na Tabela 4.

Tabela 4 - Correlação das dimensões do SF-36 com experiências religiosas/espirituais em pacientes com diagnóstico de câncer atendidos no Serviço de Emergência. São Paulo, SP, Brasil, 2017 (continua)

\begin{tabular}{lcc} 
Dimensões SF-36 & \multicolumn{2}{c}{$\begin{array}{c}\text { Escala de Experiência } \\
\text { religiosa/espiritual }\end{array}$} \\
\cline { 2 - 3 } & $\mathbf{R}^{\dagger}$ & p-valor \\
\hline Capacidade funcional & $-0,4$ & 0,000 \\
\hline Aspecto físico & $-0,22$ & 0,049 \\
\hline Dor & $-0,45$ & $<0,0001$ \\
\hline Estado geral de saúde & $-0,44$ & $<0,0001$ \\
\hline
\end{tabular}




\begin{tabular}{lcc}
\hline Vitalidade & $-0,54$ & $<0,0001$ \\
\hline Aspectos sociais & $-0,54$ & $<0,0001$ \\
\hline Aspectos emocionais & $-0,46$ & $<0,0001$ \\
\hline Saúde mental & $-0,63$ & $<0,0001$ \\
${ }^{+}$Coeficiente de Correlação de Spearman & &
\end{tabular}

\section{DISCUSSÃO}

O perfil da amostra estudada mostra predomínio de homens, com média de idade de 58 anos, religião católica e ensino fundamental. Entre os sistemas afetados pelo câncer, a maioria dos casos envolve as neoplasias do sistema reprodutor. Resultados semelhantes como idade entre 41 a 60 anos, ensino fundamental, predomínio da religião católica e neoplasia do sistema reprodutor foram encontrados nos pacientes de outro estudo cujo objetivo foi investigar inter-relações entre bem-estar espiritual, depressão e qualidade de vida durante o enfrentamento do câncer; a principal diferença foi que as mulheres compuseram a maioria da amostra ${ }^{(14)}$.

A maioria dos participantes $(79,5 \%)$ da presente pesquisa tinha comorbidades, sendo a mais prevalente a cardiopatia (69,0\%), e 97,5\% faziam uso de medicamentos, os analgésicos foram os mais citados. Esse dado pode ser explicado pelo aumento da expectativa de vida e, consequentemente, das doenças crônicas não transmissíveis como as cardiovasculares ${ }^{(15)}$. O alto uso de analgésicos pode ser explicado pelo fato de que a dor é um dos principais sintomas que acometem o paciente com câncer; no Brasil, a estimativa é que 62 a 90\% dos pacientes com câncer apresentam algum tipo de dor ${ }^{(16)}$.

A maioria dos entrevistados deste estudo respondeu ter rede de apoio, sendo que as Unidades Básicas de Saúde foram as mais citadas. Estudo com objetivo de investigar interrelações entre bem-estar espiritual, depressão e qualidade de vida durante o enfrentamento do câncer evidenciou busca significativa do paciente oncológico por espiritualidade e melhora em sua qualidade de vida, sendo que o apoio do cônjuge, demais familiares, amigos e pessoas religiosas constituem uma rede social de apoio a estes pacientes ${ }^{(14)}$. As inter-relações do indivíduo em seus diversos ambientes: família e trabalho, saúde e cultura, valores, crenças, afetam as relações. Assim, os vínculos estabelecidos na rede de apoio podem ou não potencializar o processo de desenvolvimento do indivíduo para lidar com sua doença(17).

Neste estudo, as dimensões da qualidade de vida que se mostraram mais comprometidas foram "Aspecto físico", seguida de "Estado Geral de Saúde" e "Capacidade Funcional". Do mesmo modo, estudo que avaliou a qualidade de vida de pacientes com câncer de pulmão(18) evidenciou menores escores de qualidade de vida para todos os domínios quando comparados com indivíduos saudáveis, tendo igualmente ao presente estudo, o domínio de aspectos físicos como aquele que apresentou a menor pontuação.

O domínio Aspectos físicos avalia as limitações quanto ao tipo e quantidade de trabalho, bem como o quanto essas limitações dificultam a realização do trabalho e das atividades da vida diária(19). Pode-se inf̧erir que a diminuição do valor do escore dos aspectos físicos pode estar relacionada aos sintomas decorrentes da doença e/ou tratamento, embora não tenhamos aplicado uma escala para quantificar sintomas. Esses sintomas podem trazer limitações na realização de atividades da vida diária e laborais, interferindo diretamente na qualidade de vida.

Estudo que avaliou a qualidade de vida relacionada à saúde de pacientes com câncer em cuidados paliativos, mostrou a dimensão Estado Geral de Saúde como sendo também 
uma das que obteve a pior avaliação(20).

Outros estudos ${ }^{(18,20,21)}$ corroboram a influência do câncer sobre a capacidade funcional e qualidade de vida em pacientes com câncer, com prejuízos nas atividades de vida diária, relações sociais e situação financeira.

Estudo que verificou associação entre a qualidade de vida e a capacidade funcional durante o tratamento de câncer hematológico também verificou associação entre menores scores de QV e incapacidade funcional, sendo que a fadiga foi o sintoma que se mostrou associado à incapacidade para as atividades básicas de vida diária e a insônia para as atividades instrumentais ${ }^{(22)}$.

Estudo de revisão(9) identificou sinais e sintomas como preditores de baixa QV: dor, fadiga, distúrbio do sono, depressão, alterações nutricionais, entre outros.

Tendo em vista que o câncer pode acometer qualquer órgão e que as abordagens de tratamento apresentam o potencial de efeitos multissistêmicos, a prática de enfermagem oncológica, em qualquer nível de atenção, requer conhecimento amplo de todos os sistemas de órgãos, além de uma assistência com acurada identificação de problemas. Os achados citados acima denotam que cuidados de enfermagem centrados no controle de sinais e sintomas são imprescindíveis para melhorar a qualidade de vida do paciente com câncer.

Os entrevistados desta pesquisa apresentaram uma média de escore baixa para experiências religiosas/espirituais. Este resultado merece atenção, uma vez que pessoas religiosas podem apresentar um comportamento mais saudável, já que a maioria das religiões orientam sobre hábitos saudáveis de vida ${ }^{(23)}$. A vivência religiosa e espiritual é algo que pode proporcionar um maior contato com a realidade subjetiva interna e favorecer possíveis mudanças de atitudes e ideias frente às experiências atuais da realidade de cada indivíduo ${ }^{(24)}$.

O presente estudo verificou que, quanto menor a frequência de experiências religiosas/ espirituais, pior a qualidade de vida. Outros estudos envolvendo pacientes oncológicos também demonstraram influência positiva da religiosidade e da espiritualidade, tanto na saúde física quanto na saúde mental e social e no enfrentamento da doença e agravo, ou seja, a religiosidade/espiritualidade tem influência direta na $\mathrm{VV}^{(14,25,26)}$.

Estudo transversal, realizado no Chile com 208 pacientes, avaliando a associação entre a dor espiritual e qualidade de vida em pacientes com câncer, evidenciou que menores escores na avaliação da qualidade de vida foram associados a mais jovens, com maior sofrimento físico, ansiedade ou depressão e menor religiosidade e enfrentamento religioso. $\mathrm{Na}$ análise multivariada, a QV foi independentemente associada à dor espiritual, enfrentamento religioso e sofrimento físico ${ }^{(27)}$. Esses achados podem inferir que a avaliação da religiosidade/espiritualidade pode dar subsídios para a equipe de saúde implementar ações para a melhoria da qualidade de vida.

Para pacientes oncológicos, a relação entre a doença e a possibilidade de morte fazem do enfrentamento religioso uma estratégia de redução do estresse e melhoria da qualidade de vida(28).

Como limitação do estudo, tem-sese o fato de ter sido realizado em centro único, com assistência somente prestada a pacientes do sistema público de saúde, o que pode não representar outras realidades. Outros estudos envolvendo a temática com essa população devem ser encorajados. No entanto, este estudo corrobora que o paciente oncológico, incluindo aquele atendido em situação de emergência, deve ser assistido em sua totalidade, e que as questões religiosas/espirituais devem ser consideradas pela equipe de saúde, para que sua influência positiva contribua para a qualidade de vida e para o enfrentamento da problemática vivida. 
O presente estudo evidenciou que a dimensão da QV mais comprometida foi a de "Aspecto físico", seguida de "Estado Geral de Saúde" e "Capacidade Funcional". Os entrevistados apresentaram baixa frequência de experiências religiosas/espirituais. Foi observada correlação entre QV e Experiências religiosas/espirituais para pacientes oncológicos em um serviço de emergência, sendo que quanto menor a frequência de experiências religiosas/espirituais, mais comprometida foi a QV.

Espera-se que este estudo contribua para mudanças positivas na prática profissional dos enfermeiros emergencistas. Buscar prestar cuidado seguro e humanizado aos pacientes oncológicos são aspectos importantes que precisam ser sempre almejados, bem como valorizar e respeitar a espiritualidade/religiosidade na prática assistencial, de modo que os profissionais possam contribuir para melhorar a qualidade de vida desses pacientes nos serviços de saúde.

\section{REFERÊNCIAS}

1. Instituto Nacional do Câncer José Alencar Gomes da Silva. O que é câncer? [Internet]. 2016 [acesso em 25 jan 2019]. Disponível em: http://www1.inca.gov.br/conteudo view.asp?id=322.

2. Silva PLN da, Ruas PR, Barbosa HA, Soares LM, Rocha GG da. O significado do câncer: percepção de pacientes. Rev enferm UFPE [Internet]. 2013 [acesso em 25 jan 2019]; 7(12). Disponível em: https:// periodicos.ufpe.br/revistas/revistaenfermagem/article/view/12345/15065.

3. Instituto Nacional de Câncer José Alencar Gomes da Silva. Estimativa 2016: incidência de câncer no Brasil. [Internet]. 2018 [acesso em 21 ago 2019]. Disponível em: http://www1.inca.gov.br/estimativa/2018/ estimativa-2018.pdf.

4. Guerrero GP, Zago MMF, Sawada NO, Pinto MH. Relação entre espiritualidade e câncer: perspectiva do paciente. Rev. bras. enferm. [Internet]. 2011 [acesso em 25 jan 2019]; 64(1). Disponível em: http:// www.scielo.br/pdf/reben/v64n1/v64n1a08.pdf.

5. Gabriel J. Acute oncological emergencies. Nurs Stand. [Internet]. 2012 [acesso em 25 jan 2019]; 27(4). Disponível em: https://www.ncbi.nlm.nih.gov/pubmed/23101297.

6. Sadik M, Ozlem K, Huseyin M, AliAyberk B, Ahmet S, Ozgur O. Attributes of can $\neg$ cer patients admitted to the emergency department in one year. World J Emerg Med. [Internet]. 2014 [acesso em 25 jan 2019]; 5(2). Disponível em: https://www.ncbi.nlm.nih.gov/pmc/articles/PMC4129880/.

7. Trincaus MR, Corrêa AK. A dualidade vida-morte na vivência dos pacientes com metástase. Rev Esc Enferm USP. [Internet]. 2007 [acesso em 25 jan 2019]; 41(1). Disponível em: http://www.ee.usp.br/reeusp/ upload/pdf/301.pdf.

8. Pinto AC, Marchesini SM, Zugno PI, Zimmermann KG, Dagostin KG, Soratto MT. A importância da espiritualidade em pacientes com câncer. Rev Saúde.Com. [Internet]. 2015 [acesso em 25 jan 2019]; 11(2). Disponível em: http://periodicos2.uesb.br/index.php/rsc/article/view/351.

9. Freire MEM, Sawada NO, França ISX de, Costa SFG da, Oliveira CDB. Qualidade de vida relacionada à saúde de pacientes com câncer avançado: uma revisão integrativa. Rev Esc Enferm USP. [Internet]. 2014 [acesso em 25 jan 2019]; 48(2). Disponível em: http://www.scielo.br/pdf/reeusp/v48n2/pt 0080-6234reeusp-48-02-357.pdf.

10. World Health Organization. Division of mental health and prevention of substance abuse. WHOQOL and spirituality, religiousness and personal beliefs (SRPB). Genebra: Report on WHO consultation; 1998 [acesso em 25 fev 2019]; Disponível em: https://www.who.int/mental health/media/en/622.pdf. 
11. Fornazari AS, Ferreira RER. Religiosidade/espiritualidade em pacientes oncológicos: qualidade de vida e saúde. Psic.: Teor. e Pesq. [Internet]. 2010 [acesso em 25 jan 2019]; 26(2). Disponível em: http:// www.scielo.br/pdf/ptp/v26n2/a08v26n2.pdf.

12. Ciconelli RM. Tradução para a língua portuguesa e validação do questionário genérico de avaliação de qualidade de vida SF-36 (Brasil SF-36). [tese]. São Paulo (SP): Universidade Federal de São Paulo; 1997. [acesso em 21 ago 2019]. Disponível em: http://repositorio.unifesp.br/bitstream/ handle/11600/15360/Tese-3099.pdf?sequence=1\&isAllowed=y.

13. Kimura M, Oliveira AL de, Mishima LS, Underwood LG. Adaptação cultural e validação da Underwood's Daily Spiritual Experience Scale- versão brasileira. Rev Esc Enferm USP. [Internet]. 2012 [acesso em 25 jan 2019]; 46(Esp). Disponível em: http://dx.doi.org/10.1590/S0080-62342012000700015.

14. Miranda SL de, Lanna MAL e, Felippe WC. Espiritualidade, depressão e qualidade de vida no enfrentamento do câncer: estudo exploratório. Psicol cienc prof. [Internet]. 2015 [acesso em 25 jan 2019]; 35(3). Disponível em: http://dx.doi.org/10.1590/1982-3703002342013.

15. Ministério da Saúde (BR). DATASUS. Informações de Saúde. Disponível em: http://www2.datasus.gov. br/DATASUS/index.php?area=0205\&VObj=http://tabnet.datasus.gov.br/cgi/deftohtm.exe?sim/cnv/obt10.

16. Cunha FF da, Rêgo L de P. Enfermagem diante da dor oncológica. Rev dor. [Internet]. 2015 [acesso em 25 jan 2019]; 16(2). Disponível em: http://dx.doi.org/10.5935/1806-0013.20150027.

17. Juliano MCC, Yunes MAM. Reflexões sobre rede de apoio social como mecanismo de proteção e promoção de resiliência. Ambient. soc. [Internet]. 2014 [acesso em 03 dez 2018]; 17(3):135-54. Disponível em: http://dx.doi.org/10.1590/S1414-753X2014000300009.

18. Franceschini J, Santos AA dos, Mouallem IE, Jamnik S, Uehara C, Fernandes ALG, et al. Avaliação da qualidade de vida em pacientes com câncer de pulmão através da aplicação do questionário Medical Outcomes Study 36-item Short-Form Health Survey. J. bras. pneumol. [Internet]. 2008 [acesso em 03 dez 2018]; 34(6):387-93. Disponível em: http://dx.doi.org/10.1590/S1806-37132008000600009.

19. Ware JE, Sherbourne CD. The MOS 36-item short-form health survey (SF-36) I. Conceptual framework and item selection. Med Care. [Internet]. 1992 [acesso em 02 dez 2018]; 30(6):473-83. Disponível em: https://www.ncbi.nlm.nih.gov/pubmed/1593914.

20. Freire MEM, Costa SFG da, Lima RAG de, Sawada NO. Qualidade de vida relacionada à saúde de pacientes com câncer em cuidados paliativos. Texto contexto- enferm [Internet]. 2018 [acessp e, 02 dez 2018]; 27(2):e5420016. Disponível em: http://dx.doi.org/10.1590/0104-070720180005420016.

21. Costa WA, Eleutério Jr J, Giraldo PC, Gonçalvez AK. Quality of life in breast cancer survivors. Rev. Assoc. Med. Bras. [Internet]. 2017 [acesso em 02 dez 2018]; 63(7): 583-9. Disponível em: http://dx.doi. org/10.1590/1806-9282.63.07.583.

22. Souza MV de, Christofoletti M, Streb AR, Duca GFD. Quality of life and functional capacity during the treatment of hematologic neoplasms. Fisioter. mov. [Internet]. 2018 [acesso em 02 dez 2018]; 31:e003137. Disponível em: http://dx.doi.org/10.1590/1980-5918.031.ao37.

23. Santos NC dos, Abdala GA. Religiosidade e qualidade de vida relacionada à saúde dos idosos em um município na Bahia, Brasil. Rev bras geriatr gerontol. [Internet]. 2014 [acesso em 25 jan 2019];17(4). Disponível em: http://dx.doi.org/10.1590/1809-9823.2014.13166.

24. Alves DG, Assis MR de. O desenvolvimento religioso e espiritual e a saúde mental: discutindo alguns de seus significados. Conexões PSI. [Internet]. 2015 [acesso em 25 jan 2019]; 3(1). Disponível em: http:// apl.unisuam.edu.br/revistas/index.php/conexoespsi/article/view/582/551.

25. Cheng Q, Liu X, Li X, Wang Y, Mao T, Chen Y. Improving spiritual well-being among cancer patients: implications for clinical care. Support Care Cancer. [Internet]. 2019 [acesso em 25 jan 2019]. Disponível em: https://link.springer.com/article/10.1007\%2Fs00520-019-4636-4. 
26. Maiko S, Johns SA, Helft PR, Slaven JE, Cottingham AH, Torke AM. Spiritual experiences of adults with advanced cancer in outpatient clinical settings. J Pain Symptom Manage. [Internet]. 2019 [acesso em 25 jan 2019]; 57(3). Disponível em: https://doi.org/10.1016/j.jpainsymman.2018.11.026.

27. Peres-Cruz PE, Larger P, Carrasco C, Bonati P, Batic B, Satt LT, et al. Spiritual Pain Is Associated with Decreased Quality of Life in Advanced Cancer Patients in Palliative Care: An Exploratory Study. J Palliat Med. [Internet]. 2019 [acesso em 25 jan 2019]. Disponível em: https://doi.org/10.1089/jpm.2018.0340.

28. Yoon SJ, Suh S-Y, Kim SH, Park J, Kim YJ, Kang B, et al. Spiritual well being among palliative care patients with different religious affiliations: a multicenter korean study. J Pain Symptom Manage. [Internet]. 2018 [acesso em 21 ago 2019]; 56(6). Disponível em: https://doi.org/10.1016/j. jpainsymman.2018.09.001.

Recebido: $17 / 06 / 2019$

Finalizado: 25/03/2020

Editora associada: Luciana Puchalski Kalinke

Autor Correspondente:

Carla Roberta Monteiro Miura

Universidade Federal de São Paulo

R. Alexandre Baptistone, 783 - 06190-120 - Osasco, São Paulo, Brasil

E-mail: carla.monteiro@unifesp.br

Contribuição dos autores:

Contribuições substanciais para a concepção ou desenho do estudo; ou a aquisição, análise ou interpretação de dados do estudo - CRMM, CRVC, REAB, MCBTL, PFB, MFPO

Elaboração e revisão crítica do conteúdo intelectual do estudo - CRMM, CRVC, REAB, MCBTL, PFB, MFPO

Aprovação da versão final do estudo a ser publicado - CRMM, CRVC, REAB, MCBTL, PFB, MFPO

Responsável por todos os aspectos do estudo, assegurando as questões de precisão ou integridade de qualquer parte do estudo - CRMM, CRVC, REAB, MCBTL, PFB, MFPO 